Article

\title{
Cauchy Problem for a Linear System of Ordinary Differential Equations of the Fractional Order
}

\section{Murat Mamchuev}

Institute of Applied Mathematics and Automation, Kabardin-Balkar Scientific Center of Russian Academy of Sciences, 89-A Shortanov Street, 360000 Nalchik, Russia; mamchuev@rambler.ru

Received: 28 July 2020; Accepted: 25 August 2020; Published: 1 September 2020

check for updates

\begin{abstract}
We investigate the initial problem for a linear system of ordinary differential equations with constant coefficients and with the Dzhrbashyan-Nersesyan fractional differentiation operator. The existence and uniqueness theorems of the solution of the boundary value problem under the study are proved. The solution is constructed explicitly in terms of the Mittag-Leffler function of the matrix argument. The Dzhrbashyan-Nersesyan operator is a generalization of the Riemann-Liouville, Caputo and Miller-Ross fractional differentiation operators. The obtained results as particular cases contain the results related to the study of initial problems for the systems of ordinary differential equations with Riemann-Liouville, Caputo and Miller-Ross derivatives and the investigated initial problem that generalizes them.
\end{abstract}

Keywords: fractional derivatives; Dzhrbashyan-Nersesyan fractional differentiation operator; systems of ordinary differential equations of fractional order; initial problem; conditions for unique solvability

\section{Introduction}

Consider the system of ordinary differential equations

$$
L u(x) \equiv D_{0 x}^{\left\{\alpha_{0}, \alpha_{1}, \ldots, \alpha_{m}\right\}} u(x)-A u(x)=f(x)
$$

where $D_{0 x}^{\left\{\alpha_{0}, \alpha_{1}, \ldots, \alpha_{m}\right\}}$ is the Dzhrbashyan-Nersesyan fractional differentiation operator of the order $\alpha=\sum_{i=0}^{m} \alpha_{i}-1>0[1], \alpha_{i} \in(0,1](i=\overline{0, m}) ; f(x)=\left\|f_{1}(x), f_{2}(x), \ldots, f_{n}(x)\right\|$ and $u(x)=$ $\left\|u_{1}(x), u_{2}(x), \ldots, u_{n}(x)\right\|$ are respectively given and unknown $n$-vectors and $A$ is a given constant $n \times n$ matrix.

The Dzhrbashyan-Nersesyan fractional differentiation operator $D_{s t}^{\left\{\gamma_{0}, \gamma_{1}, \ldots, \gamma_{m}\right\}}$ associated with the sequence $\left\{\gamma_{0}, \gamma_{1}, \ldots, \gamma_{m}\right\}$, of order $\gamma=\sum_{i=0}^{m} \gamma_{i}-1>0, \gamma_{i} \in(0,1],(i=\overline{0, m})$ was determined by the ratio [1]

$$
\begin{gathered}
D_{s t}^{\left\{\gamma_{0}, \gamma_{1}, \ldots, \gamma_{m}\right\}} v(t)=D_{s t}^{\gamma_{m}-1} D_{s t}^{\gamma_{m-1}} \ldots D_{s t}^{\gamma_{1}} D_{s t}^{\gamma_{0}} v(t), \\
D_{s t}^{\left\{\gamma_{0}\right\}} v(t)=D_{s t}^{\gamma_{0}-1} v(t),
\end{gathered}
$$

where $D_{s t}^{v}$ is the Riemann-Liouville fractional integro-differentiation operator of order $v$. The operator $D_{s t}^{v}$ for $v<0$ is defined as follows ([2], p. 9):

$$
D_{s t}^{v} v(t)=\frac{\operatorname{sgn}(t-s)}{\Gamma(-v)} \int_{s}^{t} \frac{v(\xi) d \xi}{|t-\xi|^{v+1}}
$$


where $\Gamma(z)$ is the Euler gamma function. For $v \geq 0$ the operator $D_{s t}^{v}$ can be determined using the recursive relation

$$
D_{s t}^{v} v(t)=\operatorname{sgn}(t-s) \frac{d}{d t} D_{s t}^{v-1} v(t) .
$$

In 1954, J.H. Barrett [3] investigated the initial problem for the equation

$$
\begin{gathered}
D_{a x}^{\alpha} y(x)+\lambda y(x)=h(x), \\
\lim _{x \rightarrow a} D_{a x}^{\alpha-i} y(x)=K_{i}, \quad i=1,2, \ldots, n, \quad n-1<\operatorname{Re}(\alpha)<n, \quad n \in \mathbb{N} .
\end{gathered}
$$

In 1968, M.M. Dzhrbashyan and A.B. Nersesyan [1] introduced the fractional differentiation operator $D_{0 t}^{\left\{\gamma_{0}, \gamma_{1}, \ldots, \gamma_{m}\right\}}$ and investigated the Cauchy problem for the Equation (1) for $n=1$.

Systems of linear ordinary equations of fractional order were first investigated in the works of V.K. Veber [4-8] and M.I. Imanaliev and V.K. Veber [5]. In 1976, V.K. Veber [4] provided the solution of the Cauchy problem for the system of equations

$$
D_{0 x}^{\alpha} y(x)=A y(x), \quad 0<\alpha \leq 2
$$

with constant matrix $A$ in terms of the Mittag-Leffler function of the matrix argument. The asymptotic behavior as $x \rightarrow \infty$ of various solutions of this system (including the fundamental matrix) was studied in $[5,6]$. Later, V.K. Veber considered the Cauchy problem for the inhomogeneous system

$$
D_{0 x}^{\alpha} y(x)=A(x) y(x)+f(x), \quad n-1<\alpha \leq n, \quad n=1,2, \ldots
$$

with continuous matrix function $A(x)$ for $x \geq 0$ [7], and in [8] he constructed a fundamental solution of this system with a constant matrix $A$ in terms of the Mittag-Leffler function of a matrix argument. There are also examples of some applications that lead to systems of equations with fractional derivatives in the paper [8].

A.A. Chikriy and I.I. Matychyn in $[9,10]$ obtained solutions of Cauchy problems for the systems of equations of the form (1), with Riemann-Liouville, Caputo and Miller-Ross derivatives using the Laplace transform.

In the works [11,12] I. Matychyn and V. Onyshchenko investigated the solutions of the initial problems for systems of equations with fractional Riemann-Liouville and Caputo derivatives analytically and numerically using the Mittag-Leffler matrix function.

Note that $[13,14]$ investigated boundary value problems for multidimensional systems of partial differential equations of fractional order. In [15] attention was drawn to the fact that in the one-dimensional case these results coincide with the results of [4].

In this paper, we investigate the initial problem for the system (1). We prove some properties of the Mittag-Leffler matrix function, obtain a general representation of the solutions to the system (1) and prove the theorem of the unique solvability of the Cauchy problem for this system.

\section{Statement of the Problem and the Solvability Theorem}

Problem 1. Find a solution $u(x)$ of the system (1) in the interval $(0, l)$, with the conditions

$$
\lim _{x \rightarrow 0} D_{0 x}^{\left\{\alpha_{0}, \alpha_{1}, \ldots, \alpha_{k}\right\}} u(x)=u_{0}^{k}, \quad 0 \leq k \leq m-1,
$$

where $u_{0}^{k}=\left\|u_{1}^{k}, u_{2}^{k}, \ldots, u_{n}^{k}\right\|$ given real constant $n$-vectors.

A solution $u(x)$ of the system (1) such that $D_{0 x}^{\left\{\alpha_{0}, \alpha_{1}, \ldots, \alpha_{m}\right\}} u(x) \in C(0, l), D_{0 x}^{\left\{\alpha_{0}, \alpha_{1}, \ldots, \alpha_{k}\right\}} u(x) \in$ $C^{1}(0, l) \cup C[0, l](k=\overline{0, m-1})$ is called a regular solution of the system (1) in the interval $(0, l)$. 
Theorem 1. Let $\alpha_{0}+\alpha_{m}>1$, function $f(x) \in C(0, l)$ can be presented as $f(x)=D_{0 x}^{\alpha_{m}-1} f_{0}(x)$, where $f_{0}(x) \in L(0, l)$. Then there exists a unique regular in the interval $(0, l)$ solution of Problem 1 . Solution can be represented as

$$
u(x)=\sum_{i=1}^{m} D_{0 x}^{\left\{\alpha_{m}, \alpha_{m-1}, \ldots, \alpha_{m+1-i}\right\}} G(x) u_{0}^{m-i}+\int_{0}^{x} G(x-t) f(t) d t,
$$

where $G(x)=x^{\alpha-1} E_{\alpha, \alpha}\left(A x^{\alpha}\right)$.

\section{Preliminaries}

The formula

$$
\int_{0}^{x} h(t) D_{0 t}^{v} g(t) d t=\int_{0}^{x} g(t) D_{x t}^{v} h(t) d t, \quad v<0
$$

is known as the formula of fractional integration by parts ([2], p. 9).

The following formula

$$
\begin{gathered}
D_{0 x}^{v} \int_{0}^{x} h(x-t) g(t) d t=\int_{0}^{x} h(x-t) D_{0 t}^{v} g(t) d t+ \\
\quad+h(x) \lim _{t \rightarrow 0} D_{0 t}^{v-1} g(t), \quad 0<v<1
\end{gathered}
$$

holds ([16], p. 99).

The following formula of fractional integro-differentiation of power function

$$
D_{a x}^{v} \frac{|x-a|^{\mu-1}}{\Gamma(\mu)}=\frac{|x-a|^{\mu-v-1}}{\Gamma(\mu-v)}
$$

is valid for $\mu>0$ if $v \in \mathbb{R}$, and for $\mu \in \mathbb{R}$ if $v \in \mathbb{N}$.

In 1903, Mittag-Leffler introduced the function [17] $E_{\alpha}(z)$

$$
E_{\alpha}(z)=\sum_{k=0}^{\infty} \frac{z^{k}}{\Gamma(\alpha k+1)}, \quad(\alpha \in \mathbb{C} ; \operatorname{Re}(\alpha)>0),
$$

which is now known as the Mittag-Leffler function.

In 1905, A. Wiman [18] generalized this function with the two-parameter Mittag-Leffler function $E_{\alpha, \beta}(z)$ (also sometimes called the Wiman function)

$$
E_{\alpha, \beta}(z)=\sum_{k=0}^{\infty} \frac{z^{k}}{\Gamma(\alpha k+\beta)}, \quad(\alpha, \beta \in \mathbb{C} ; \operatorname{Re}(\alpha)>0, \operatorname{Re}(\beta)>0) .
$$

The following properties of this function are valid:

$$
\begin{gathered}
E_{\alpha, \beta}(z)=\frac{1}{\Gamma(\beta)}+z E_{\alpha, \beta+\alpha}(z), \\
D_{0 x}^{\mu} x^{\beta-1} E_{\alpha, \beta}\left(\lambda x^{\alpha}\right)=x^{\beta-\mu-1} E_{\alpha, \beta-\mu}\left(\lambda x^{\alpha}\right), \quad(\beta>0, \mu \in \mathbb{R}) .
\end{gathered}
$$

In 1971, an even more general function $E_{\alpha, \beta}^{\gamma}(z)$ was introduced by T.R. Prabhakar [19]

$$
E_{\alpha, \beta}^{\gamma}(z)=\sum_{k=0}^{\infty} \frac{(\gamma)_{k}}{\Gamma(\alpha k+\beta)} \frac{z^{k}}{k !}, \quad(\alpha, \beta, \gamma \in \mathbb{C} ; \operatorname{Re}(\alpha)>0, \operatorname{Re}(\beta)>0, \operatorname{Re}(\gamma)>0),
$$


where $(\gamma)_{k}=\frac{\Gamma(\gamma+k)}{\Gamma(\gamma)}$ is the Pohhammer symbol. This function is known as the Prabhakar function.

The following equalities hold [19-21]:

$$
\begin{gathered}
E_{\alpha, \beta}^{1}(z)=E_{\alpha, \beta}(z), \\
E_{\alpha, \beta}^{\gamma}(z)=E_{\alpha, \beta}^{\gamma-1}(z)+z E_{\alpha, \beta+\alpha}^{\gamma}(z), \quad(\operatorname{Re}(\alpha)>0, \beta>0, \gamma \in \mathbb{C}), \\
D_{0 x}^{\mu} x^{\beta-1} E_{\alpha, \beta}^{\gamma}\left(\lambda x^{\alpha}\right)=x^{\beta-\mu-1} E_{\alpha, \beta-\mu}\left(\lambda x^{\alpha}\right), \quad(\beta>0, \mu \in \mathbb{R}), \\
\lim _{z \rightarrow 0} E_{\alpha, \beta}^{\gamma}(z)=\frac{1}{\Gamma(\beta)} .
\end{gathered}
$$

In 1976, V.K. Veber [4] introduced the Mittag-Leffler function of the matrix argument (see also [11]). Note that in the paper [4] the function $E_{\alpha, \beta}^{\gamma}(z)$ with natural values of the parameter $\gamma \in \mathbb{N}$ naturally comes into sight.

Here we give a definition of the Mittag-Leffler function of a matrix argument and then examine some of its properties.

Let $A$ be a square matrix and $H$ a matrix reducing the matrix $A$ to Jordan matrix i.e.,

$$
A=H J H^{-1}=H\left[J_{r_{1}}\left(\lambda_{1}\right), J_{r_{2}}\left(\lambda_{2}\right), \ldots, J_{r_{p}}\left(\lambda_{p}\right)\right] H^{-1},
$$

where $J(\lambda)=\operatorname{diag}\left[J_{1}\left(\lambda_{1}\right), \ldots, J_{p}\left(\lambda_{p}\right)\right]$ is a quasi-diagonal matrix with blocks of the form

$$
J_{k} \equiv J_{k}\left(\lambda_{k}\right)=\left\|\begin{array}{cccc}
\lambda_{k} & 1 & \ldots & 0 \\
& \lambda_{k} & \ldots & 0 \\
& 0 & \ddots & \vdots \\
& & & \lambda_{k}
\end{array}\right\|, \quad k=1, \ldots, p,
$$

$\lambda_{1}, \ldots, \lambda_{p}$ are the eigenvalues of the matrix $A, J_{k}\left(\lambda_{k}\right)$ are square matrices of order $r_{k}+1, \sum_{k=1}^{p} r_{k}+p=n$.

Then we have

$$
E_{\alpha, \beta}(A z)=H E_{\alpha, \beta}(J z) H^{-1}=H \operatorname{diag}\left[E_{\alpha, \beta}\left[J_{1}\left(\lambda_{1}\right) z\right], \ldots, E_{\alpha, \beta}\left[J_{p}\left(\lambda_{p}\right)\right]\right] H^{-1},
$$

where

$$
\begin{gathered}
E_{\alpha, \beta}\left[J_{k}(\lambda) z\right]=\left\|\begin{array}{cccc}
e_{0} & e_{1} & \ldots & e_{r_{k}-1} \\
& e_{0} & \ldots & e_{r_{k}-2} \\
& 0 & \ddots & \vdots \\
& & e_{0}
\end{array}\right\|, \\
e_{n} \equiv e_{n}^{\alpha, \beta}(\lambda, z)=z^{n} \sum_{k=0}^{\infty} \frac{(k+n) !}{k ! n !} \frac{\lambda^{k} z^{k}}{\Gamma(\alpha(k+n)+\beta)}=z^{n} E_{\alpha, \alpha n+\beta}^{n+1}(\lambda z) .
\end{gathered}
$$

It's obvious that

$$
e_{0} \equiv e_{0}^{\alpha, \beta}(\lambda, z)=E_{\alpha, \beta}(\lambda z) .
$$

\section{Properties of the Mittag-Leffler Function of a Matrix Argument}

1. The following equality holds:

$$
E_{\alpha, \beta}(A z)=\frac{1}{\Gamma(\beta)} I+A z E_{\alpha, \beta+\alpha}(A z), \quad(\beta>0, \mu \in \mathbb{R}) .
$$


Indeed, by the relation (8), we obtain

$$
e_{0}^{\alpha, \beta}(\lambda, z)=E_{\alpha, \beta}(\lambda z)=\frac{1}{\Gamma(\beta)}+\lambda z E_{\alpha, \beta+\alpha}(\lambda z)=\frac{1}{\Gamma(\beta)}+\lambda z e_{0}^{\alpha, \beta+\alpha}(\lambda, z) .
$$

For $n \neq 0$, taking into account the relation (11), we have

$$
\begin{gathered}
e_{n}^{\alpha, \beta}(\lambda, z)=z^{n} E_{\alpha, \alpha n+\beta}^{n+1}(\lambda z)=z^{n} E_{\alpha, \alpha n+\beta}^{n}(\lambda z)+\lambda z^{n+1} E_{\alpha, \alpha n+\beta+\alpha}^{n+1}(\lambda z)= \\
=z e_{n}^{\alpha, \beta+\alpha}(\lambda, z)+\lambda z e_{n+1}^{\alpha, \beta+\alpha}(\lambda, z) .
\end{gathered}
$$

From the last equalities we obtain

$$
\begin{aligned}
& \left\|\begin{array}{cccl}
e_{0} & e_{1} & \ldots & e_{r_{k}-1} \\
& e_{0} & \ldots & e_{r_{k}-2} \\
& 0 & \ddots & \vdots \\
& & & e_{0}
\end{array}\right\|=\frac{1}{\Gamma(\beta)} I+z\left\|\begin{array}{ccll}
\lambda \bar{e}_{0} & \bar{e}_{1}+\lambda \bar{e}_{1} & \ldots & \bar{e}_{r_{k}-1}+\lambda \bar{e}_{r_{k}-2} \\
& \lambda \bar{e}_{0} & \ldots & \bar{e}_{r_{k}-2}+\lambda \bar{e}_{r_{k}}-2 \\
& 0 & \ddots & \vdots \\
& & & \lambda \bar{e}_{0}
\end{array}\right\|= \\
& =\frac{1}{\Gamma(\beta)} I+z\left\|\begin{array}{|cccl}
\lambda_{k} & 1 & \ldots & 0 \\
& \lambda_{k} & \ldots & 0 \\
& 0 & \ddots & \vdots \\
& & & \lambda_{k}
\end{array}\right\|\left\|\begin{array}{cccl}
\bar{e}_{0} & \bar{e}_{1} & \ldots & \bar{e}_{r_{k}-1} \\
& \bar{e}_{0} & \ldots & \bar{e}_{r_{k}-2} \\
& 0 & \ddots & \vdots \\
& & & \bar{e}_{0}
\end{array}\right\| \text {, }
\end{aligned}
$$

where $\bar{e}_{n} \equiv e_{n}^{\alpha, \beta+\alpha}(\lambda, z)=z^{n} E_{\alpha, \alpha n+\alpha+\beta}^{n+1}(\lambda z)$. Thus,

$$
E_{\alpha, \beta}\left[J_{r_{k}}\left(\lambda_{k}\right) z\right]=\frac{1}{\Gamma(\beta)} I+J_{r_{k}}\left(\lambda_{k}\right) z E_{\alpha, \beta+\alpha}\left[J_{r_{k}}\left(\lambda_{k}\right) z\right] .
$$

Hence, by virtue of (14), the equality (15) follows.

2. For the matrix function an analogue of the formula (9) of fractional integro-differentiation is valid

$$
D_{0 x}^{\mu} x^{\beta-1} E_{\alpha, \beta}\left(A x^{\alpha}\right)=x^{\beta-\mu-1} E_{\alpha, \beta-\mu}\left(A x^{\alpha}\right), \quad(\beta>0, \mu \in \mathbb{R}) .
$$

Indeed, by virtue of the equality (12) we have

$$
\begin{aligned}
& D_{0 x}^{\mu} x^{\beta-1} E_{\alpha, \beta}\left[J_{r_{k}}\left(\lambda_{k}\right) z\right]=D_{0 x}^{\mu} x^{\beta-1}\left\|\begin{array}{cccc}
e_{0} & e_{1} & \ldots & e_{r_{k}-1} \\
& e_{0} & \ldots & e_{r_{k}-2} \\
& 0 & \ddots & \vdots \\
& & & e_{0}
\end{array}\right\|= \\
& =x^{\beta-\mu-1}\left\|\begin{array}{cccc}
\tilde{e}_{0} & \tilde{e}_{1} & \ldots & \tilde{e}_{r_{k}-1} \\
& \tilde{e}_{0} & \ldots & \tilde{e}_{r_{k}-2} \\
0 & \ddots & \vdots \\
& & \tilde{e}_{0}
\end{array}\right\|=x^{\beta-\mu-1} E_{\alpha, \beta-\mu}\left[J_{r_{k}}\left(\lambda_{k}\right) z\right],
\end{aligned}
$$

where $\tilde{e}_{n} \equiv e_{n}^{\alpha, \beta-\mu}(\lambda, z)=z^{n} E_{\alpha, \alpha n+\beta-\mu}^{n+1}(\lambda z)$. Taking into account (14) we get (16).

3. From (15) and (16) it follows

$$
\left(D_{0 x}^{\alpha}-A\right) x^{\beta-1} E_{\alpha, \beta}\left(A x^{\alpha}\right)=\frac{x^{\beta-\alpha-1}}{\Gamma(\beta-\alpha)} I .
$$

4. Let us act on the function $x^{\beta-1} E_{\alpha, \beta}\left(A x^{\alpha}\right)$ by the Dzhrbashyan-Nersesyan operator. 
We denote $\mu_{j}=\sum_{i=0}^{j} \gamma_{i}$. Then, by virtue of formula (16), for $\beta-\mu_{j} \geq 0$ we get the following equality

$$
D_{0 x}^{\left\{\gamma_{0}, \gamma_{1}, \ldots, \gamma_{j}\right\}} x^{\beta-1} E_{\alpha, \beta}\left(A x^{\alpha}\right)=x^{\beta-\mu_{j}} E_{\alpha, \beta-\mu_{j}+1}\left(A x^{\alpha}\right) .
$$

In particular for $\beta-\mu_{j}=0$ we have

$$
D_{0 x}^{\left\{\gamma_{0}, \gamma_{1}, \ldots, \gamma_{j}\right\}} x^{\beta-1} E_{\alpha, \beta}\left(A x^{\alpha}\right)=E_{\alpha, 1}\left(A x^{\alpha}\right) .
$$

By using the formula (15) from (18) we obtain

$$
D_{0 x}^{\left\{\gamma_{0}, \gamma_{1}, \ldots, \gamma_{j}\right\}} x^{\beta-1} E_{\alpha, \beta}\left(A x^{\alpha}\right)=\frac{x^{\beta-\mu_{j}}}{\Gamma\left(\beta-\mu_{j}+1\right)} I+A x^{\beta+\alpha-\mu_{j}} E_{\alpha, \beta+\alpha-\mu_{j}+1}\left(A x^{\alpha}\right)
$$

for $\beta-\mu_{j} \geq 0$.

From the last equality for $j=m$ and $\gamma_{i}=\alpha_{i}(i=\overline{0, m})$, we get

$$
\left(D_{0 x}^{\left\{\alpha_{0}, \ldots, \alpha_{m}\right\}}-A\right) x^{\beta-1} E_{\alpha, \beta}\left(A x^{\alpha}\right)=\frac{x^{\beta-\alpha-1}}{\Gamma(\beta-\alpha)} I, \quad \beta \geq \mu_{m}=\alpha+1,
$$

and for $j=m$ and $\gamma_{i}=\alpha_{m-i}(i=\overline{0, m})$, we get

$$
\left(D_{0 x}^{\left\{\alpha_{m}, \ldots, \alpha_{0}\right\}}-A\right) x^{\alpha} E_{\alpha, \alpha+1}\left(A x^{\alpha}\right)=I .
$$

5. By virtue of relation (13) we obtain

$$
\lim _{z \rightarrow 0} e_{n}^{\alpha, \beta}(\lambda, z)= \begin{cases}0, & n>0 \\ \frac{1}{\Gamma(\beta)}, & n=0 .\end{cases}
$$

From the last formula and (14) we get

$$
\lim _{x \rightarrow 0} E_{\alpha, \beta}\left(A x^{\alpha}\right)=\frac{1}{\Gamma(\beta)} I,
$$

and

$$
\lim _{x \rightarrow 0} x^{\beta-1} E_{\alpha, \beta}\left(A x^{\alpha}\right)= \begin{cases}0, & \beta>1, \\ I, & \beta=1 .\end{cases}
$$

\section{Green's Formula for the Operator $L$}

Lemma 1. Let $f(x) \in L(0, l)$, then every regular solution to Problem 1 can be represented by formula (3).

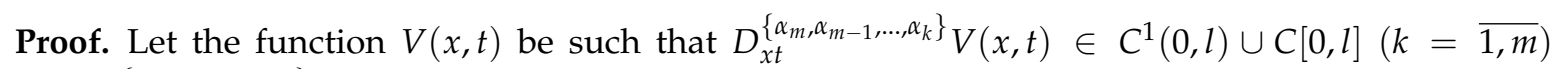
and $D_{x t}^{\left\{\alpha_{m}, \alpha_{m-1}, \ldots, \alpha_{0}\right\}} V(x, t) \in C(0, l) \cup L(0, l)$ for any fixed $x \in[0, l]$. Using the definitions of the Dzhrbashyan-Nersesyan and Riemann-Liouville operators, the integration by parts formula and the formula (4), we transform the following integral 


$$
\begin{gathered}
\int_{0}^{x} V(x, t) D_{0 t}^{\left\{\alpha_{0}, \alpha_{1}, \ldots, \alpha_{m}\right\}} u(t) d t=\int_{0}^{x} V(x, t) D_{0 t}^{\alpha_{m}-1} \frac{d}{d t} D_{0 t}^{\left\{\alpha_{0}, \alpha_{1}, \ldots, \alpha_{m-1}\right\}} u(t) d t= \\
=\int_{0}^{x} D_{x t}^{\alpha_{m}-1} V(x, t) \cdot \frac{d}{d t} D_{0 t}^{\left\{\alpha_{0}, \alpha_{1}, \ldots, \alpha_{m-1}\right\}} u(t) d t=S_{1}(x)-\int_{0}^{x} \frac{d}{d t} D_{x t}^{\alpha_{m}-1} V(x, t) \cdot D_{0 t}^{\left\{\alpha_{0}, \alpha_{1}, \ldots, \alpha_{m-1}\right\}} u(t) d t= \\
=S_{1}(x)+\int_{0}^{x} D_{x t}^{\alpha_{m}} V(x, t) \cdot D_{0 t}^{\alpha_{m-1}-1} \frac{d}{d t} D_{0 t}^{\left\{\alpha_{0}, \alpha_{1}, \ldots, \alpha_{m-2}\right\}} u(t) d t= \\
=S_{1}(x)+\int_{0}^{x} D_{x t}^{\alpha_{m-1}-1} D_{x t}^{\alpha_{m}} V(x, t) \cdot \frac{d}{d t} D_{0 t}^{\left\{\alpha_{0}, \alpha_{1}, \ldots, \alpha_{m-2}\right\}} u(t) d t= \\
=S_{1}(x)+\int_{0}^{x} D_{x t}^{\left\{\alpha_{m}, \alpha_{m-1}\right\}} V(x, t) \cdot \frac{d}{d t} D_{0 t}^{\left\{\alpha_{0}, \alpha_{1}, \ldots, \alpha_{m-2}\right\}} u(t) d t
\end{gathered}
$$

where

$$
S_{1}(x)=\left.D_{x t}^{\left\{\alpha_{m}\right\}} V(x, t) \cdot D_{0 t}^{\left\{\alpha_{0}, \alpha_{1}, \ldots, \alpha_{m-1}\right\}} u(t)\right|_{t=0} ^{t=x} .
$$

Continuing similarly, we obtain

$$
\begin{gathered}
\int_{0}^{x} V(x, t) D_{0 t}^{\left\{\alpha_{0}, \alpha_{1}, \ldots, \alpha_{m}\right\}} u(t) d t=\left.\sum_{i=1}^{k} D_{x t}^{\left\{\alpha_{m}, \alpha_{m-1}, \ldots, \alpha_{m+1-i}\right\}} V(x, t) \cdot D_{0 t}^{\left\{\alpha_{0}, \alpha_{1}, \ldots, \alpha_{m-i}\right\}} u(t)\right|_{t=0} ^{t=x}+ \\
+\int_{0}^{x} D_{x t}^{\left\{\alpha_{m}, \alpha_{m-1}, \ldots, \alpha_{m-k}\right\}} V(x, t) \cdot \frac{d}{d t} D_{0 t}^{\left\{\alpha_{0}, \alpha_{1}, \ldots, \alpha_{m-k-1}\right\}} u(t) d t=\ldots= \\
=\left.\sum_{i=1}^{m} D_{x t}^{\left\{\alpha_{m}, \alpha_{m-1}, \ldots, \alpha_{m+1-i}\right\}} V(x, t) \cdot D_{0 t}^{\left\{\alpha_{0}, \alpha_{1}, \ldots, \alpha_{m-i}\right\}} u(t)\right|_{t=0} ^{t=x}+\int_{0}^{x} D_{x t}^{\left\{\alpha_{m}, \alpha_{m-1}, \ldots, \alpha_{0}\right\}} V(x, t) \cdot u(t) d t .
\end{gathered}
$$

Thus, the formula

$$
\begin{gathered}
\int_{0}^{x}\left[V(x, t) D_{0 t}^{\left\{\alpha_{0}, \alpha_{1}, \ldots, \alpha_{m}\right\}} u(t)-D_{x t}^{\left\{\alpha_{m}, \alpha_{m-1}, \ldots, \alpha_{0}\right\}} V(x, t) \cdot u(t)\right] d t= \\
=\left.\sum_{i=1}^{m} D_{x t}^{\left\{\alpha_{m}, \alpha_{m-1}, \ldots, \alpha_{m+1-i}\right\}} V(x, t) \cdot D_{0 t}^{\left\{\alpha_{0}, \alpha_{1}, \ldots, \alpha_{m-i}\right\}} u(t)\right|_{t=0} ^{t=x}
\end{gathered}
$$

holds.

Let the function $V(x, t)$ be a solution of the equation

$$
L^{*} V(x, t) \equiv D_{x t}^{\left\{\alpha_{m}, \alpha_{m-1}, \ldots, \alpha_{0}\right\}} V(x, t)-V(x, t) A=I,
$$

and satisfy the following conditions

$$
\lim _{t \rightarrow x} D_{x t}^{\left\{\alpha_{m}, \alpha_{m-1}, \ldots, \alpha_{m+1-i}\right\}} V(x, t)=0, \quad 1 \leq i \leq m .
$$

From the formulas (21) and (22) and

$$
D_{x t}^{\gamma} v(x-t)=\left.D_{0 y}^{\gamma} v(y)\right|_{y=x-t}
$$


it follows that the function

$$
V(x, t)=(x-t)^{\alpha} E_{\alpha, \alpha+1}\left(A(x-t)^{\alpha}\right)
$$

is the solution of the problem (23), (24).

From (1) and (23), it follows that

$$
V(x, t) L u(t)-L^{*} V(x, t) \cdot u(t)=V(x, t) f(t)-u(t) .
$$

After integrating the equality (26) we obtain

$$
\left.\sum_{i=1}^{m} D_{x t}^{\left\{\alpha_{m}, \alpha_{m-1}, \ldots, \alpha_{m+1-i}\right\}} V(x, t) \cdot D_{0 t}^{\left\{\alpha_{0}, \alpha_{1}, \ldots, \alpha_{m-i}\right\}} u(t)\right|_{t=0} ^{t=x}=\int_{0}^{x} V(x, t) f(t) d t-\int_{0}^{x} u(t) d t .
$$

Using (4) and (24) from the equality (27) we get

$$
\int_{0}^{x} u(t) d t=\int_{0}^{x} V(x, t) f(t) d t+\left.\sum_{i=1}^{m} D_{x t}^{\left\{\alpha_{m}, \alpha_{m-1}, \ldots, \alpha_{m+1-i}\right\}} V(x, t)\right|_{t=0} u_{0}^{m-i} .
$$

Differentiating (28) and taking into account the equality $V(x, x)=0$ we obtain

$$
u(x)=\int_{0}^{x} G(x, t) f(t) d t+\left.\sum_{j=0}^{m-1} D_{x t}^{\left\{\alpha_{m}, \alpha_{m-1}, \ldots, \alpha_{j+1}\right\}} G(x, t)\right|_{t=0} u_{0}^{j},
$$

where $G(x, t)=V_{x}(x, t)=G(x-t)$,

$$
G(x)=x^{\alpha-1} E_{\alpha, \alpha}\left(A x^{\alpha}\right) .
$$

Applying to (29) the formula

$$
\lim _{t \rightarrow 0} D_{x t}^{\gamma} v(x-t)=D_{0 x}^{\gamma} v(x),
$$

that follows from (25), we get the equality (3).

\section{Proof of Theorem 1}

Proof. Let us prove that the function (3) is the solution of Problem 1. We denote the last term in right hand side of (3) as $u_{f}(x)$ and $u_{C}(x)=u(x)-u_{f}(x)$.

By virtue of (18) we get

$$
D_{0 x}^{\left\{\alpha_{m}, \ldots, \alpha_{k}\right\}} G(x)=x^{\alpha-v_{k}} E_{\alpha, \alpha-v_{k}+1}\left(A x^{\alpha}\right)=x^{\mu_{k-1}-1} E_{\alpha, \mu_{k-1}}\left(A x^{\alpha}\right),
$$

where $\mu_{k}=\sum_{i=0}^{k} \alpha_{i}, v_{k}=\sum_{i=k}^{m} \alpha_{i}$.

Using the following equalities

$$
\alpha-\mu_{k}-v_{s}+1= \begin{cases}\alpha_{s+1}+\ldots+\alpha_{k-1}, & s<k-1 \\ 0, & s=k-1 \\ -\left(\alpha_{k}+\ldots+\alpha_{s}\right), & s>k-1\end{cases}
$$


the formulas (15), (18) and (30) we obtain

$$
D_{0 x}^{\left\{\alpha_{0}, \ldots, \alpha_{s}\right\}} D_{0 x}^{\left\{\alpha_{m}, \ldots, \alpha_{k}\right\}} G(x)= \begin{cases}x^{\alpha_{s+1}+\ldots+\alpha_{k-1}} E_{\alpha, \alpha_{s+1}+\ldots+\alpha_{k-1}+1}\left(A x^{\alpha}\right), & s<k-1, \\ E_{\alpha, 1}\left(A x^{\alpha}\right), & s=k-1, \\ A x^{\mu_{k-1}+v_{s+1}-1} E_{\alpha, \mu_{k-1}+v_{s+1}}\left(A x^{\alpha}\right), & s>k-1 .\end{cases}
$$

From (31), (22) and the inequality $\alpha_{0}+\alpha_{m}>1$ it follows that

$$
\lim _{x \rightarrow 0} D_{0 x}^{\left\{\alpha_{0}, \ldots, \alpha_{s}\right\}} D_{0 x}^{\left\{\alpha_{m}, \ldots, \alpha_{k}\right\}} G(x)= \begin{cases}0, & s<k-1, \\ I, & s=k-1, \\ 0, & s>k-1 .\end{cases}
$$

Equality (32) gives the relations

$$
\lim _{x \rightarrow 0} D_{0 x}^{\left\{\alpha_{m}, \ldots, \alpha_{k}\right\}} u_{C}(x)=u_{0}^{k}, \quad k=\overline{0, m-1} .
$$

For $s=m$ from (21) and (31) we get

$$
\left(D_{0 x}^{\left\{\alpha_{0}, \ldots, \alpha_{m}\right\}}-A\right) D_{0 x}^{\left\{\alpha_{m}, \ldots, \alpha_{k}\right\}} G(x)=0 .
$$

Thus, we have to show that the last term in (3) is a solution to Problem 1 with homogeneous conditions. By virtue of the condition $f(x)=D_{0 x}^{\alpha_{m}-1} f_{0}(x), f_{0}(x) \in L(0, l)$ and formulas (4) and (16) we can conclude

$$
u_{f}(x)=\int_{0}^{x} G(x-t) D_{0 x}^{\alpha_{m}-1} f_{0}(t) d t=\int_{0}^{x} G_{0}(x-t) f_{0}(t) d t
$$

where

$$
G_{0}(x)=D_{0 x}^{\alpha_{m}-1} G(x)=x^{\mu_{m-1}-1} E_{\alpha, \mu_{m-1}}\left(A x^{\alpha}\right) .
$$

The last equality yields

$$
D_{0 x}^{\left\{\alpha_{0}\right\}} u_{f}(x)=\int_{0}^{x} D_{x t}^{\alpha_{0}-1} G_{0}(x-t) \cdot f_{0}(t) d t .
$$

Using relation (18) we get

$$
D_{0 x}^{\left\{\alpha_{0}, \ldots, \alpha_{k}\right\}} G_{0}(x)=x^{\mu_{m-1}-\mu_{k}} E_{\alpha, \mu_{m-1}-\mu_{k}+1}\left(A x^{\alpha}\right) .
$$

By virtue of (5), (16) and (22) we have

$$
\begin{aligned}
& D_{0 x}^{\alpha_{0}} u_{f}(x)=\frac{d}{d x} D_{0 x}^{\left\{\alpha_{0}\right\}} u_{f}(x)=\int_{0}^{x} D_{x t}^{\alpha_{0}} G_{0}(x-t) \cdot f_{0}(t) d t+ \\
& +\left(\lim _{t \rightarrow x} D_{0 x}^{\alpha_{0}-1} G_{0}(x-t)\right) f_{0}(x)=\int_{0}^{x} D_{x t}^{\alpha_{0}} G_{0}(x-t) \cdot f_{0}(t) d t .
\end{aligned}
$$

Continuing in a similar way we obtain the following equalities

$$
D_{0 x}^{\left\{\alpha_{0}, \ldots, \alpha_{k}\right\}} u_{f}(x)=\int_{0}^{x} D_{x t}^{\left\{\alpha_{0}, \ldots, \alpha_{k}\right\}} G_{0}(x-t) f_{0}(t) d t
$$


and

$$
\begin{gathered}
\frac{d}{d x} D_{0 x}^{\left\{\alpha_{0}, \ldots, \alpha_{k}\right\}} u_{f}(x)=\int_{0}^{x} \frac{d}{d x} D_{0 x}^{\left\{\alpha_{0}, \ldots, \alpha_{k}\right\}} G_{0}(x-t) f_{0}(t) d t+ \\
+\left(\lim _{t \rightarrow x} D_{x t}^{\left\{\alpha_{0}, \ldots, \alpha_{k}\right\}} G_{0}(x-t)\right) f_{0}(x)=\int_{0}^{x} \frac{d}{d x} D_{0 x}^{\left\{\alpha_{0}, \ldots, \alpha_{k}\right\}} G_{0}(x-t) f_{0}(t) d t .
\end{gathered}
$$

For $k=m-1$ from (36) we get

$$
D_{0 x}^{\left\{\alpha_{0}, \ldots, \alpha_{m-1}\right\}} u_{f}(x)=\int_{0}^{x} E_{\alpha, 1}\left(A(x-t)^{\alpha}\right) f_{0}(t) d t .
$$

From the relation (37) using (15) we get

$$
\begin{gathered}
\frac{d}{d x} D_{0 x}^{\left\{\alpha_{0}, \ldots, \alpha_{m-1}\right\}} u_{f}(x)=\frac{d}{d x} \int_{0}^{x}(x-t)^{\alpha} E_{\alpha, \alpha+1}\left(A(x-t)^{\alpha}\right) f_{0}(t) d t= \\
=\lim _{t \rightarrow x} E_{\alpha, 1}\left(A(x-t)^{\alpha}\right) f_{0}(t)+\int_{0}^{x}(x-t)^{\alpha-1} E_{\alpha, \alpha}\left(A(x-t)^{\alpha}\right) f_{0}(t) d t= \\
=f_{0}(x)+A \int_{0}^{x} G(x-t) f_{0}(t) d t .
\end{gathered}
$$

From the last relation, it follows that

$$
\begin{gathered}
D_{0 x}^{\left\{\alpha_{0}, \ldots, \alpha_{m}\right\}} u_{f}(x)=D_{0 x}^{\left\{\alpha_{m}\right\}} \frac{d}{d x} D_{0 x}^{\left\{\alpha_{0}, \ldots, \alpha_{m}-1\right\}} u_{f}(x)= \\
=D_{0 x}^{\alpha_{m}-1}\left(f_{0}(x)+A \int_{0}^{x}(x-t)^{\alpha-1} E_{\alpha, \alpha}\left(A(x-t)^{\alpha}\right) f_{0}(t) d t\right)= \\
=f(x)+A \int_{0}^{x} G(x-t) f(t) d t=f(x)+A u_{f}(x) .
\end{gathered}
$$

Equalities (35) and (36) lead to the relation

$$
\lim _{x \rightarrow 0} D_{0 x}^{\left\{\alpha_{0}, \ldots, \alpha_{k}\right\}} u_{f}(x)=0, \quad 0 \leq k \leq m-1 .
$$

The relations (33), (34), (38) and (39) mean that the function (3) is the solution to Problem 1. The uniqueness of the solution to Problem 1 follows from Lemma 1.

\section{Conclusions}

The article investigates the initial problem for a linear system of ordinary differential equations with the Djrbashyan-Nersesyan fractional differentiation operator with constant coefficients. To solve the problem under the study, the Green's function method is implemented, an integral representation of the solution is obtained, the properties of the matrix Mittag-Leffler function (autotransformation formula, fractional integro-differentiation formulas, etc.) are investigated. The results obtained can be used to study local and nonlocal boundary value problems for system (1).

Funding: This research received no external funding.

Conflicts of Interest: The author declares no conflict of interest. 


\section{References}

1. Dzrbashyan, M.M.; Nersesyan, A.B. Fractional derivatives and the Cauchy problem for fractional differential equations. Izv. Acad. Sci. Arm. SSR Mat. 1968, 3, 3-28.

2. Nakhushev, A.M. Fractional Calculus and Its Application; Fizmatlit: Moscow, Russia, 2003.

3. Barrett, J.H. Differential Equations of Non-Integer Order. Can. J. Math. 1954, 6, 529-541. [CrossRef]

4. Veber, V.K. The structure of general solution of the system $y^{(\alpha)}=A y, 0<\alpha \leq 1$. Trudy Kirgiz. Gos. Univ. Ser. Mat. Nauk 1976, 11, 26-32.

5. Imanaliev, M.I.; Veber, V.K. On a generalization of a function of Mittag-Leffler type and its application. In Issledovaniya Po Integro-Differentsial'nym Uravneniyam v Kirgizii; Ilim: Frunze, Russia, 1980; Volume 13, pp. 49-59.

6. Veber, V.K. Asymptotic behavior of solutions of a linear system of differential equations of fractional order. In Issledovaniya Po Integro-Differentsial'nym Uravneniyam v Kirgizii; llim: Frunze, Russia, 1983; Volume 16, pp. 119-125.

7. Veber, V.K. On the general theory of linear systems with fractional derivatives. In Issledovaniya Po Integro-Differentsial'nym Uravneniyam v Kirgizii; Ilim: Frunze, Russia, 1985; Volume 18, pp. 301-305.

8. Veber V.K. Linear equations with fractional derivatives and constant coefficients in spaces of generalized functions. In Issledovaniya Po Integro-Differentsial'nym Uravneniyam v Kirgizii; Ilim: Frunze, Russia, 1985; Volume 18, pp. 306-312.

9. Chikriy, A.A.; Matichin, I.I. On an analogue of the Cauchy formula for linear systems of any fractional order. Rep. Nation. Ac. Sci. Ukr. 2007, 1, 53-55.

10. Chikriy, A.A.; Matichin, I.I. Presentation of Solutions of Linear Systems with Fractional Derivatives in the Sense of Riemann-Liouville, Caputo, and Miller-Ross. J. Autom. Inf. Sci. 2008, 40, 1-11.

11. Matychyn, I.; Onyshchenko, V. Optimal control of linear systems with fractional derivatives. Fract. Calc. Appl. Anal. 2018, 21, 134-150. [CrossRef]

12. Matychyn, I.; Onyshchenko, V. Matrix Mittag-Leffler function in fractional systems and its computation. Bull. Pol. Acad. Tech. 2018, 66, 495-500.

13. Mamchuev, M.O. Boundary value problem for a system of multidimensional differential equations of fractional order. Vest. Samara State Univ. Nat. Sci. Ser. 2008, 8/2, 164-175.

14. Mamchuev, M.O. Boundary value problem for a multidimensional system of equations with RiemannLiouville fractional derivatives. Siberian Electron. Math. Rep. 2019, 16, 732-747.

15. Mamchuev, M.O. Boundary Value Problems for Equations and Systems of Equations with the Partial Derivatives of Fractional Order; Publishing house KBSC of RAS: Nalchik, Russia, 2013.

16. Podlubny, I. Fractional Differential Equations; Academtic Press: New York, NY, USA, 1999.

17. Mittag-Leffler, G.M. Sur la nouvelle fonction $E_{\alpha}(x)$. C. R. Acad. Sci. Paris 1903, 137, 554-558.

18. Wiman, A. Über den fundamental Satz in der Theorie der Funktionen $E_{\alpha}(x)$. Acta Math. 1905, $29,191-201$. [CrossRef]

19. Prabhakar, T.R. A singular integral equation with a generalized Mittag-Leffler function in the kernel. Yokohama Math. J. 1971, $19,7-15$.

20. Shukla, A.K.; Prajapati, J.C. On a generalization of Mittag-Leffler function and its properties. J. Math. Anal. Appl. 2007, 336, 797-811. [CrossRef]

21. Povstenko, Y. Linear Fractional Diffusion-Wave Equation for Scientists and Engineers; Birkhäuser: New York, NY, USA, 2015.

(C) 2020 by the author. Licensee MDPI, Basel, Switzerland. This article is an open access article distributed under the terms and conditions of the Creative Commons Attribution (CC BY) license (http:/ / creativecommons.org/licenses/by/4.0/). 